\title{
Reflexiones en comunidad de práctica sobre Triángulos imposibles en clase de matemáticas
}

\author{
Luis Alexander Conde Solano' \\ Sandra Evely Parada Rico" \\ Jorge Enrique Fiallo Leal"I
}

\section{Resumen}

En este artículo presentamos resultados de una investigación desarrollada en una comunidad de práctica de educadores matemáticos que incorporan tecnologías digitales en sus prácticas profesionales en el contexto colombiano. El estudio se centra en la problemática del uso de la tecnología para la enseñanza de las matemáticas y el uso restringido de ésta, lo que impide un verdadero impacto en el aprendizaje del área. Al respecto, se plantea como objetivo general de la investigación: describir significados negociados (en términos de aprendizajes) en una comunidad de práctica de educadores matemáticos que incorporan las tecnologías digitales en sus prácticas profesionales. La investigación sigue una metodología cualitativa tipificada como investigación acción colaborativa. En los resultados hallados, destacamos experiencias con profesores de educación básica secundaria que han trabajado bajo el modelo metodológico de Reflexión-y-Acción. Dicho modelo gira alrededor de los procesos de reflexión-para-la acción, reflexión-en-la acción y reflexión-sobre-la acción de profesores que participan en la comunidad de práctica. Rescatamos aquí las reflexiones de profesores sobre la construcción de ángulos con el apoyo de tecnología en una clase de matemáticas con estudiantes de octavo grado. Podemos concluir que el conjunto de procesos reflexivos da cuenta de cómo puede cambiar el rumbo de la clase planeada por el profesor, a consecuencia de los recursos didácticos concretos o virtuales que selecciona y usa para promover actividad matemática por parte de sus estudiantes.

\section{Palabras clave:}

Comunidad de práctica - Procesos de reflexión - Formación de profesores - Tecnologías digitales.

I- Universidad de Medellín, Medellín, Colombia. Contacto: Iconde@udem.edu.co II- Universidad Industrial de Santander, Bucaramanga, Colombia. Contacto: sparada@ matematicas.uis.edu.co

III- Universidad Industrial de Santander, Bucaramanga, Colombia. Contacto: jfiallo@ uis.edu.co 


\title{
Reflections in a community of practice on impossible triangles in mathematics classes
}

Luis Alexander Conde Solano'

Sandra Evely Parada Rico"

Jorge Enrique Fiallo Leal"II

\begin{abstract}
This paper presents results of an investigation developed in a community of practice of mathematics educators who incorporate digital technologies in their professional practices in Colombia. The study concentrates on the problematics of the use of technology for mathematics teaching and its restricted use, which prevents real impact on mathematics learning. In this regard, the research's general objective is: to describe negotiated meanings (in terms of learning) in a community of practice of mathematics educators that incorporate digital technology in their professional practices. The investigation follows a qualitative methodology called collaborative action research. As for findings, we emphasized experiences with teachers of secondary education who have worked with the methodological model of Reflection-andAction. This model revolves around the processes of reflection in action, reflection-for action, and reflection-on the action of the teachers who participate in the community of practice. We focused on teachers' reflections on building angles with the support of technology in a mathematics class with eighth graders. We concluded that the set of reflective processes reveals how the course of the class planned by teachers can change as a result of the concrete or virtual teaching resources that they select and use to promote mathematics activities for their students.
\end{abstract}

\section{Keywords}

Community of practice - Reflection - Teacher education -Digital technologies.

\footnotetext{
I- Universidad de Medellín, Medellín, Colombia. Contacto: Iconde@udem.edu.co II- Universidad Industrial de Santander, Bucaramanga, Colombia.

Contacto: sparada@matematicas.uis.edu.co

III- Universidad Industrial de Santander, Bucaramanga, Colombia.

Contacto: jfiallo@uis.edu.co
} 


\section{Introducción}

Tanto la diversidad de recursos que se encuentran en la Internet, así como la constante información recibida de investigadores exhortando a los profesores a utilizar dichos recursos. Puede comprometer a los profesores a incorporar al aula, material que en vez de favorecer la actividad matemática los desvíe de los objetivos de aprendizaje. Actualmente se ha invitado a los profesores a voltear la mirada hacia el uso de las tecnologías digitales, pues se menciona que éstas favorecen el aprendizaje de los estudiantes. También se ha incrementado la sugerencia de éstas en los documentos oficiales y de la empresa privada, lo cual a muchos profesores les ocasiona temor y a otros inquietud sobre los beneficios y efectos que las tecnologías digitales puedan tener sobre sus prácticas en el aula, pero ¿se les prepara a los profesores a seleccionar, diseñar y usar dichos recursos en sus clases de matemáticas?

El Ministerio de Educación Nacional (MEN) de Colombia ha hecho esfuerzos por atender las anteriores inquietudes de los profesores en los programas de actualización docente. En este marco se realizó un proyecto de uso de tecnología en la clase de matemáticas, dotando de calculadoras a los colegios participantes y realizando cursos de formación para los profesores, de quienes se esperaba que difundieran esa formación a sus colegas en los colegios. Sin embargo, en la actualidad se puede observar que los profesores formados siguen utilizando de manera esporádica la tecnología en sus clases, y en algunos colegios se han archivado las calculadoras.

Al respecto Parada (2011) afirma que la formación profesional de los profesores, en la mayoría de los programas de desarrollo profesional (diplomados, especializaciones, maestrías) para atender sus necesidades actuales, encuentra limitaciones por tiempo, programas y evaluaciones. Es por ello que muchos programas formales de desarrollo profesional dejan ciertos vacíos, tanto de conocimientos de la matemática escolar, como de conocimientos pedagógicos y didácticos, por lo que no logran sostener y satisfacer las necesidades reales de los profesores.

En el contexto de la anterior problemática, identificamos la necesidad de generar espacios donde los profesores exploren las bondades y limitaciones que se puede tener al incorporar tecnologías digitales en la clase de matemáticas. Así mismo, para que se reflexione sobre cuándo, qué, dónde y cómo implementarlas, según los objetivos de aprendizaje previstos. En esta dirección Parada (2011) menciona que la conformación de comunidades de práctica de educadores matemáticos puede ser una posibilidad para fomentar el uso de las tecnologías digitales. A partir de esta iniciativa nos proponemos aportar herramientas a los profesores desde lo teórico, lo práctico y lo metodológico, desde la conformación e interacción al interior de una comunidad de práctica con el objetivo de describir significados negociados (en términos de aprendizajes) en una comunidad de práctica de educadores matemáticos que incorporan las tecnologias digitales en sus prácticas profesionales.

Para la consecución de dicho objetivo de investigación se propone utilizar el modelo teórico R-y-A (Reflexión-y-Acción) de Parada (2011) con el cual se aportan herramientas conceptuales y metodológicas para que el profesor reflexione sobre sus prácticas pedagógicas antes (reflexión-para-la acción), durante (reflexión-en-la acción) y después de la clase (reflexión-sobre-la acción) en el interior de comunidades de práctica de educadores matemáticos, con el fin que cada uno, desde los participantes, aporte desde sus conocimientos y experiencias herramientas necesarias que se concreticen en acciones en la clase de matemáticas. El modelo R-y-A busca incentivar el trabajo colaborativo al interior de comunidades de práctica ( $\mathrm{CoP}$ ) como espacios permanentes y favorables para la reflexión sobre la práctica docente y por ende para el mejoramiento de su desempeño en el aula. 


\section{Aspectos teóricos}

El modelo Reflexión-y-Acción (R-y-A) propuesto por Parada (2011) pretende constituirse en una herramienta que oriente los procesos de reflexión de los profesores. En la Figura 1 mostramos un bosquejo de dicho modelo metodológico, que tiene una lectura del exterior al centro. En las secciones siguientes se definirán cada uno de los elementos que lo componen. El anillo exterior describe los procesos que se realizan en el interior de las comunidades de educadores matemáticos (profesores, autoridades educativas, investigadores e investigadores en formación); el modelo se fundamenta en los procesos que son posibles cuando los educadores matemáticos se unen para desarrollar un pensamiento reflexivo sobre el área.

Figura 1 - Bosquejo del modelo teórico aplicado

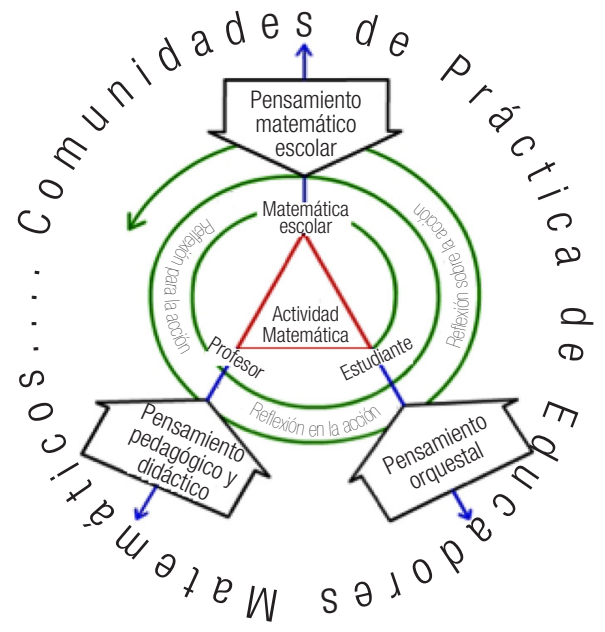

Fuente: Construcción propia

Dicho modelo está enmarcado en la participación de educadores matemático al interior de una Comunidad de Práctica (CoP). Para Wenger (1998), una CoP es un grupo de personas que comparten una preocupación, un conjunto de problemas o un interés común acerca de un tema, y que profundizan su conocimiento en esta área a través de una estructura social basada en la construcción colaborativa de conocimientos a beneficio de todos sus miembros. Wenger (1998) fija tres premisas que caracterizan una CoP: 1) el compromiso mutuo, 2) la empresa conjunta y 3) el repertorio compartido.

En nuestro estudio, enfocamos el análisis de los datos desde la perspectiva del desarrollo de un repertorio compartido, con el cual los profesores tienen la oportunidad de participar en la actividad matemática propuesta. El análisis de la participación en el repertorio de prácticas nos proporciona elementos para caracterizar cómo una comunidad de práctica puede influir en el desarrollo profesional de los profesores.

\section{Pensamiento reflexivo del profesor de matemáticas}

Las tres flechas que están alrededor de la espiral dan cuenta de los tres aspectos sobre los cuales se propone el desarrollo del pensamiento reflexivo de los profesores de matemáticas. El pensamiento, según Vega (1990), es una actividad global del sistema cognitivo que ocurre siempre que nos enfrentamos a una tarea o problema, con un objetivo y un cierto nivel de incertidumbre sobre la forma de realizarla. Es por ello que en el modelo se rescata como idea esencial que la formación de profesores no debe apuntar a un cúmulo de conocimientos, sino más bien al desarrollo de un pensamiento reflexivo, en el que se privilegien los saberes adquiridos por cada profesor en el trayecto de su práctica $\mathrm{y}$ en las maneras como usen dichos saberes para resolver los problemas cognitivos, didácticos, tecnológicos, sociales y de otro tipo, que suelen darse en el aula. No obstante, reconocemos la complejidad de la labor y por ello, como ya se ha mencionado, descomponemos dicho pensamiento en tres partes:

\section{Pensamiento matemático escolar}

Shulman (1987) enfatiza que, para enseñar, en primer lugar, hay que comprender críticamente un conjunto de ideas que van a enseñarse. Se espera que el profesor entienda lo que enseña $y$, cuando sea posible, que lo 
haga de diversas maneras. Además, necesita comprender el modo en que una determinada idea se relaciona con otras ideas al interior de la misma materia y también con ideas de otras materias. Nosotros concordamos con varios de los puntos antes mencionados; pero antes queremos considerar una diferencia entre pensamiento matemático y conocimiento matemático, para explicar que, más allá de interesarnos en que el profesor sea un experto en el área y que comprenda los contenidos curriculares, nos interesa que pueda usar sus conocimientos matemáticos para conducir la actividad matemática de los estudiantes de acuerdo a los objetivos de aprendizaje previstos. De igual forma, concebimos el pensamiento geométrico como una forma de pensamiento matemático. Jungk (1982) y Rizo (1987), asumen el pensamiento geométrico como una forma de pensar ante situaciones que requieren de los conocimientos, habilidades y capacidades geométricas y que potencia el desarrollo de ese pensamiento general y único de cada persona.

En este caso nos interesa la actividad en geometría que el profesor promueve entre sus estudiantes: la que se evidencia al reconocer formas, comparar elementos observados estableciendo relaciones entre ellos y descubrir propiedades para elaborar conclusiones para formular una solución a la situación presentada.

\section{Pensamiento didáctico de la matemática escolar}

Los conocimientos didácticos
han intentado caracterizarse en varias
investigaciones, entre ellas Shulman (1986),
Llinares yrainer (2006), entre otros. Al respecto
Geddis y Wood (1997) estudian la problemática
de la transformación del conocimiento a ser
enseñado y dicen: "los productos finales de
las transformaciones pedagógicas son las
representaciones del contenido y las estrategias
de instrucción que se ponen en juego en los
encuentros de instrucción específicos" (p.
612). Wood (2008) presenta algunos elementos
teóricos que describen el conocimiento que los

profesores necesitan para mejorar el aprendizaje de sus estudiantes, conocimientos que según el autor se requieren en la organización de la instrucción y evaluación.

Desde nuestra perspectiva, el pensamiento didáctico del profesor de matemáticas se da cuando éste se cuestiona sobre las diferentes maneras de acercar los conocimientos matemáticos a los estudiantes, buscando las formas más útiles de representar los contenidos mediante analogías, ilustraciones, ejemplos, explicaciones, y demostraciones que permitan hacerla más comprensible a los alumnos. Para esto, el profesor necesita tener claridad en su pensamiento matemático escolar con el objeto de guiar a sus estudiantes hacia la actividad matemática esperada.

Ball, Hill y Bass (2005) destacan la necesidad de trabajar las relaciones entre el conocimiento matemático y pedagógico, en la formación de docentes de matemáticas. No obstante, estos elementos siempre han carecido de una integración entre sí, y de conexión de éstos con la realidad en las aulas y del ambiente profesional. Shulman (1987) plantea que se puede discernir que la base de conocimientos para la enseñanza está en el encuentro entre la materia y la pedagogía, en la capacidad de un docente para que después de comprender el contenido de la materia consiga transformarlo a través de: la planeación de clase, la adaptación de los contenidos matemáticos a las características del grupo, la elección de la metodología de trabajo en clase y de los procesos de evaluación, entre otros aspectos.

\section{Pensamiento orquestal}

Inspirados por Trouche (2004) y su idea de orquestación, planteamos como metáfora la idea de que el profesor necesita hacer las veces de conducir su clase como lo hace el director de una orquesta y que cada clase pudiera ser un concierto. Aunque es claro que en la orquesta se supone el dominio del instrumento por cada músico, lo cual es una situación que es muy diferente a la 
de una clase pues no todos los estudiantes tienen un dominio de sus saberes matemáticos y de los recursos (como es el caso de las tecnologías digitales) que se vinculan en la clase.

Caracterizamos el pensamiento orquestal del profesor de matemáticas en torno a la conducción de su clase, y en torno a las maneras como usa los recursos que ha seleccionado, de acuerdo a la actividad matemática que tiene prevista para sus estudiantes. Este pensamiento se ve reflejado únicamente durante la clase (reflexión-en-la acción) en los pensamientos matemáticos escolares. Es así como en este proceso de reflexión el pensamiento matemático y el didáctico, se convierten en un recurso más de la clase.

Dentro de nuestro modelo, proponemos que una manera de contribuir con el desarrollo del pensamiento orquestal del profesor, no es presentándole una diversidad de recursos, sino apoyándolos para que ellos puedan discernir y pensar sobre el cómo, el cuándo y el dónde usar tal o cual recurso, dependiendo de los propósitos de aprendizaje que deseen lograrse.

Es probable que durante la clase (reflexiónen-la-acción), por diferentes circunstancias, se desvíe de los propósitos establecidos para la misma. En el marco del modelo planteamos que el profesor mediante su práctica e interacción alcanza cierto grado de improvisación necesaria para reorientar la actividad matemática en el instante de la clase. En esta dirección, retomamos de la música la idea de improvisación considerada como una intervención espontánea a través del canto o ejecución instrumental atendiendo a pautas o regla claramente definidas. La improvisación es el producto del bagaje musical donde confluyen todos los conocimientos (rítmicos, melódicos, armónicos) con las experiencias anteriores del artista.

Recurrimos como metáfora la idea de que el profesor necesita realizar improvisaciones durante la marcha de la clase para superar situaciones no previstas en ella. Esta improvisación la entendemos como una intervención consciente de argumentos válidos que el profesor usa para orientar nuevamente el curso de la clase o tener la situación bajo control.
Para ello el profesor requiere un amplio bagaje matemático donde convergen su pensamiento matemático escolar, su pensamiento pedagógico y didáctico.

\section{Procesos de reflexión}

Además de los tres aspectos o perspectivas anteriores, también consideramos tres procesos de reflexión y los representamos en un recorrido en forma de espiral, como procesos de participación-reflexión-acción. Los tres procesos son: a) reflexión-para-la acción, la cual se hace presente en la relación de la matemática escolar y el profesor, cuando el profesor planifica la actividad matemática esperada por parte de los estudiantes en la clase; b) reflexión-en-la acción, la cual se hace presente en la clase y se desarrolla en los intercambios entre el profesor y los estudiantes en torno al contenido matemático de estudio y; c) reflexión-sobre-la acción, la cual se da después de la clase cuando el profesor evalúa la actividad matemática que había planeado comparada con la actividad matemática que logró, y se inicia otra vuelta en espiral.

Consideramos que cada experiencia vivida deja saberes particulares que posibilitan procesos consecuentes más sólidos, y que se transforman en la optimización de la actividad matemática, tanto de los profesores, como de los estudiantes.

\section{Actividad matemática}

En el centro del modelo (ver Figura 1), se ubica la actividad matemática que surge del triángulo pedagógico descrito por Saint-Onge (1997, apud IBÁÑEZ, 2007). En nuestro estudio, retomamos las ideas de Chevallard, Bosch y Gascón (1997) para caracterizar la actividad matemática y decir que, hacer matemáticas, es un trabajo del pensamiento que construye conceptos para resolver problemas. Estos autores describen tres grandes tipos de actividades que podrían considerarse matemáticas: 
a) utilizar matemáticas conocidas: el primer gran tipo de actividad matemática consiste en resolver problemas a partir de las herramientas matemáticas que uno ya conoce y sabe cómo utilizar;

b) aprender y enseñar matemáticas, frente a un problema que no se sabe cómo resolver;

c) crear matemáticas nuevas: en principio, se podría decir que sólo los matemáticos producen matemáticas nuevas, pero en realidad, en el nivel de los alumnos se puede afirmar que todo aquel que aprende matemáticas participa de alguna manera en un trabajo creador.

La matemática como actividad de resolución de problemas introduce en muchos casos una componente fundamental: la matematización. Matematizar, según Treffers (1987), es organizar y estructurar la información que aparece en un problema, identificando los aspectos matemáticos relevantes, descubriendo regularidades, relaciones y estructuras.

Con este modelo orientado al desarrollo profesional, se pretende que sea el profesor quien, en primera instancia, despliegue una actividad matemática, para que tenga claridad de lo que va a promover en clase por parte de los estudiantes y cómo va a lograr que los estudiantes transiten por los diferentes niveles de matematización.

\section{Aspectos metodológicos}

Esta investigación sigue una metodología cualitativa, la cual se podría tipificar como investigación acción colaborativa, dado que más allá de estudiar la problemática antes descrita se espera provocar cambios positivos de la misma. También, tuvo características de índole descriptiva, puesto que generó informes narrativos a partir la participación en la comunidad de práctica y experiencias en la investigación de campo, ya que los procesos de reflexión-en-la acción se desarrollaron en los lugares de trabajo de los profesores participantes. Para el logro de este estudio se llevaron las siguientes fases.

\section{Caracterización de la comunidad de Práctica}

Los inicios de la comunidad se hacen mediante invitación a las instituciones y profesores que trabajan de manera conjunta con alguno de los subgrupos del Grupo de Investigación en Educación Matemática de la Universidad Industrial de Santander (Edumat-UIS), así mismo se les extiende la invitación a algunos miembros de la Escuela de Matemáticas como estudiantes y profesores cátedra interesados en el fenómeno de estudio propuesto. Por lo tanto, a esta altura del proceso la Comunidad de Práctica es constituida por veinticinco participantes: diecisiete profesores del departamento de Santander, un estudiante de Licenciatura en Matemáticas, dos estudiantes de postgrado en Educación Matemática, dos profesores de cátedra, un investigador del grupo y dos profesores-investigadores de la Escuela de Matemáticas.

\section{Diseño del trabajo colaborativo}

Con el propósito de promover la interacción entre participantes de la Comunidad de Práctica de forma presencial y a distancia se dispone de un sitio Web donde los participantes tienen herramientas para el desarrollo profesional como un repositorio de materiales compartidos por profesor o investigadores, así como enlaces a páginas Web de matemática educativa y acceso a software gratuito. Otras herramientas del sitio Web se orientan a espacios de comunicación como foros de discusión y wikis, allí los participantes pueden socializar sus inquietudes y necesidades. Como complemento se programan sesiones presenciales dos viernes de cada mes, en el horario de cuatro a seis de la tarde, de acuerdo a los calendarios y horarios escolares de los participantes.

\section{Procesos de participación}

Inicialmente se trataron algunas sesiones de discusión de temas alrededor del uso de las 
tecnologías digitales en clase de matemáticas, esto con el fin de conocer la dinámica de trabajo y los procesos de negociación de significados que vienen realizando los profesores con sus colegas en las instituciones. Luego se dio a conocer el modelo R-y-A y se discutieron ejemplos de reflexión-y-acción en otras comunidades, además se analizaron cómo han sido los procesos de incorporación de las tecnologías digitales en la educación en otros contextos.

\section{Seguimiento y acompañamiento}

En esta fase podemos dar cuenta de los usos que los profesores participantes de la Comunidad de Práctica han hecho de las tecnologías digitales en sus clases de matemáticas, vislumbrando, tal vez, una interpretación sobre cómo dichos procesos han influido en el desarrollo del pensamiento de sus estudiantes.

Observamos un tránsito de los participantes de la comunidad desde sus inicios, evidenciando en sus opiniones sobre cómo conciben las tecnologías digitales en sus clases de matemáticas, pasando por negociación de significados al respecto y finalizando con una aplicación de tecnologías digitales en sus clases de matemáticas. Creemos pertinente que para medir el impacto de la incorporación efectiva de las tecnologías digitales en los estudiantes se requiere de un trabajo constante inmerso en un diálogo dentro de la Comunidad de Práctica.

\section{Análisis de experiencias}

Se les propuso desarrollar un proceso de reflexión-y-acción, es decir que planearan una clase sobre un tema que estuviera trabajando en ese momento, en la que vincularan las tecnologías digitales (reflexión-para-la acción), que las llevaran a cabo y se filmaran (reflexiónen-la acción), y finalmente que socializaran su experiencia en una sesión presencial con la Comunidad de Práctica (reflexión-sobrela acción). Los temas propuestos por los participantes fueron: a) el concepto de área de los cuadriláteros para estudiantes de séptimo grado con el uso de GeoGebra, b) Suma de expresiones algebraicas para estudiantes de octavo grado implementando el software Almuset, c) propiedades de los determinantes para estudiantes de primer semestre universitario con apoyo de Matlab, y d) el tema construcción de ángulos para estudiantes de octavo grado con el apoyo de Logo ${ }^{1}$. Este último, lo ahondaremos como caso de estudio en los párrafos siguientes.

\section{Aplicación del modelo en un caso de estudio}

En este apartado se presenta un caso de estudio que transita por los diferentes momentos de los procesos de reflexión alrededor de una clase sobre construcción de ángulos para estudiantes de octavo grado con el apoyo de Logo. Para el análisis retomamos episodios de sesiones de video grabadas correspondientes a cada proceso de reflexión. Estos episodios son apoyados por imágenes originales de los recursos utilizados por el caso de estudio.

El caso de estudio seleccionado se trata de una profesora (seudónimo: Mary) que lleva a cabo su labor docente en octavo grado de una institución pública del sector urbano de Bucaramanga, Santander. Para la profesora es importante implementar las tecnologías digitales en sus clases de matemáticas para favorecer procesos de enseñanza y aprendizaje de las matemáticas en sus estudiantes.

\section{Reflexión-para-la acción}

Desde los procesos de reflexión-para-la acción la profesora Mary junto a su compañera diseñan una planeación para luego implementarla en el aula. Al respecto Mary expone:

Primero fue una planeación, se las mostré y la hice diferente al colegio pues allá no llevamos la planeación. La misma guía

1- Logo es un lenguaje de programación de alto nivel diseñado con fines didácticos muy usado para trabajar con niños y jóvenes. 
es importantísima, nosotras detectamos que si no se hace una buena guía que sea entendible, de buena asimilación para los estudiantes, queda muy difícil también, así se vaya apoyar uno de la tecnología. En general pues eso nos dejó una enseñanza de que tenemos que planear y prever muchas cosas. (Episodio 1 - Fragmento de las entrevistas realizadas para este estudio).

Indudablemente el ejercicio de planear generó expectativa en las profesoras, esto se evidencia en el deseo de explorar diferentes programas computacionales para el trabajo de sus actividades propuestas. La planeación colectiva tuvo una influencia en las prácticas de las profesoras, ya que al considerar la incorporación de la tecnología en sus clases las condujo a la primera experiencia de reflexión, tanto en su pensamiento matemático escolar, como en su pensamiento pedagógico y didáctico. Consideramos que las profesoras se percataron de que la incorporación de recursos tecnológicos a la clase, necesita ser acorde con el contenido matemático de estudio y adaptada a los propósitos de aprendizaje previstos.

Desde el pensamiento didáctico, las profesoras consideraron el diseño de una guía para que los estudiantes se familiaricen con el software Logo. Según Mary, ni la docente ni los estudiantes conocen el programa, por lo tanto, se planteó una primera actividad para familiarizarse con la herramienta, conocer su uso y explorar los principales comandos que se utilizan para programar los movimientos de la tortuga. Para tal fin la profesora tomó la unidad 1 de EMAT ${ }^{2}$ llamada conociendo a Logo, que consiste en dar a la tortuga instrucciones para que ella realice los movimientos de: avance (AV), retrocede (RE), gira derecha (GD), gira izquierda (GI), borrar pantalla (BP), sube lápiz (SL), baja lápiz (BL), oculta tortuga (OT) y muestra tortuga (MT).

En la segunda sesión, se diseña un taller para desarrollar la temática escogida

2- EMAT, Enseñanza de las Matemáticas con Tecnología. p. 1920. Disponible en: <http://www.matedu.cinvestav. mx/ asacristan/ Actividades_Logo.pdf>. Acceso: 15 de abril de 2014. con el uso de Logo. Éste se compone en dos partes: la primera corresponde al taller donde la profesora coloca información sobre la construcción y medida de ángulos, ángulos adyacentes, ángulos consecutivos, ángulos complementarios y suplementarios.

Figura 2. Actividad de EMAT

Escribe un procedimiento que dibuje la siguiente figura

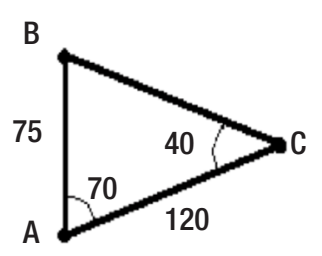

Fuente: Imagen retomada de EMAT

En la segunda parte, se proponen actividades de la unidad 17 de EMAT, que consisten en utilizar criterios de ángulos para que la tortuga ejecute giros para construir polígonos. Retomamos literalmente una de las actividades de la unidad 17 que la profesora le propone a sus estudiantes (Figura 2).

En la Figura 2, los estudiantes deben escribir un procedimiento que dibuje la figura. Para ello deben determinar medidas que en la figura no se muestran. En ella se observa que los ángulos internos del triángulo corresponden a $70^{\circ}, 40^{\circ}$ y $70^{\circ}$, cuyas medidas de los lados deberían ser 75, 120 y 120 que corresponden a un triángulo isósceles. Con este tipo de ejercicios Mary quiere enfatizarles a sus estudiantes sobre el reconocimiento de los vértices, ángulos internos y externos, ángulos suplementarios y complementarios.

\section{Reflexión-en-la acción}

La planeación fue aplicada por la profesora Mary en el aula de informática de la institución, a un grupo de 35 estudiantes de octavo grado con edades entre 12 y 14 años. El grupo se organiza en equipos de dos o tres 
integrantes por computador y cada grupo se apoya en su guía escrita para el desarrollo de las actividades. La profesora constantemente circula por la sala atendiendo las inquietudes de sus estudiantes.

En este momento de la reflexión-en-la acción, centramos la atención en un diálogo entre los estudiantes y la profesora sobre la actividad referida en la Figura 3:

Figura 3. Solución con medidas estabelecidas

\section{AV 75, GD 110, AV 120, GD 140, AV 120}

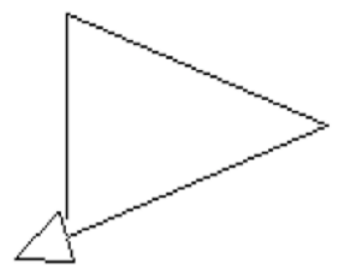

Fuente: construcción de los estudiantes en Logo

Profesora: Vaya señalándome en la pantalla cómo fueron qué hicieron

Estudiantes: Primero avanzamos 75

Profesora: Después, ¿qué hicieron?

Estudiantes: Giramos a la derecha $110^{\circ}$ porque el ángulo es de $70^{\circ}$ y $110^{\circ}+70^{\circ}=$ $180^{\circ}$, luego avanzamos 120

Profesora: ¿Por qué hicieron ese giro de $140^{\circ}$ ?

Estudiantes: No, de $143^{\circ}$

Estudiantes: Porque si avanzábamos 140 se pasaba, no alcanzaba. (Episodio 2 Fragmento de vídeo grabación de la clase)

Al construir la figura en Logo con las medidas sugeridas en la actividad de la guía, se observa que no se alcanza a cerrar el triángulo. Esta situación fue identificada por los estudiantes y desestabilizó a la profesora, pues es una actividad que proviene de un material que seguramente no requiere verificarse antes de la clase.
Figura 4. Solución con medidas estabelecidas por los

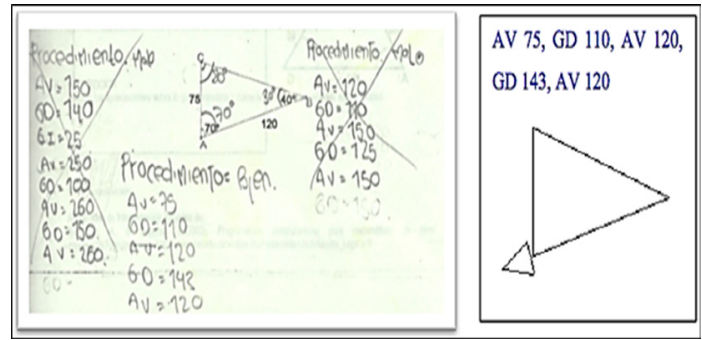

Fuente: Imagen retomada de las hojas de trabajo y construcción en logo por parte de los estudiantes

En el diálogo anterior, los estudiantes señalan (Figura 4) los ángulos internos del triángulo que corresponden a $70^{\circ}, 37^{\circ}$ y $70^{\circ}$ para poder cerrarlo, sin embargo, observamos que la suma de los ángulos internos del triángulo es diferente de $180^{\circ}$.

Esto contradice el criterio que la suma de los ángulos internos de un triángulo es de $180^{\circ}$. Si queremos un ángulo de $37^{\circ}$ entre los lados con la misma medida, también se modificarían los otros ángulos junto con los lados de la misma medida. Podemos verificar lo anterior con el apoyo de GeoGebra ${ }^{3}$ que en este caso nos ofrece una representación más detallada como la que se observa en la Figura 5.

Figura 5 Construcción en GeoGebra escala

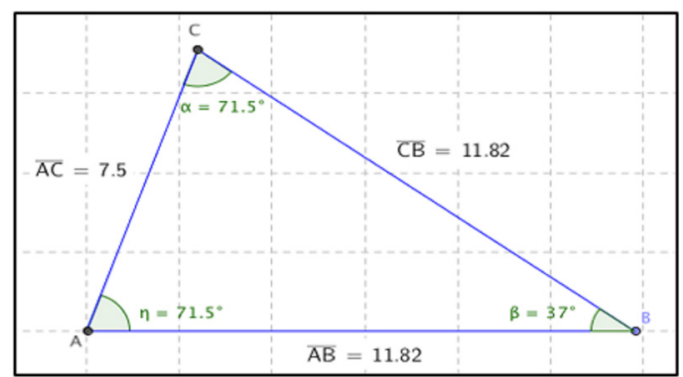

Fuente: Interpretación propia

3- GeoGebra, es un compendio de matemática con software interactivo que reúne geometría, álgebra y cálculo. 
Ahora, si queremos conservar la longitud de los lados del triángulo inicial $(75,120 \mathrm{y}$ 120). Una representación a escala (7.5, 12 y 12 unidades) en GeoGebra se aprecia un triángulo isósceles que conserva la longitud de sus lados, pero la medida de sus ángulos se modifica (Figura 6).

Figura 6. Triángulo isósceles en GeoGebra

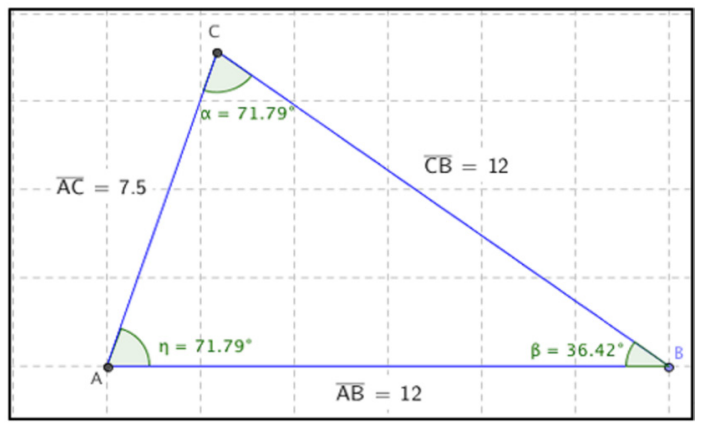

Fuente: Interpretación propia

La situación se complejiza ya que la figura propuesta en la guía difiere de la construcción hecha en Logo. Este hecho desencadena una serie de escenarios, por supuesto no contemplados en la planeación. Se genera actividad matemática por parte de los estudiantes al reconocer criterios geométricos que debían cumplir la construcción por realizar y cuyo resultado no concordaba con sus criterios. Esto se evidencia en los diálogos entre los estudiantes y Mary sobre la construcción de la figura por medio de criterios de ángulos suplementarios. Luego, se observó la inquietud por reconstruir la figura para que esta fuese una figura cerrada y además un triángulo isósceles sugerido implícitamente por las medidas establecidas en el ejercicio original.

Sin embargo, la clase finaliza con un acuerdo entre los estudiantes y la profesora sobre la solución del triángulo isósceles, cuyas medidas de sus ángulos son $70^{\circ}, 37^{\circ}$ y $70^{\circ}$ mientras que sus lados corresponden a 75, 120 y 120, situación ya comentada. De acuerdo a las evidencias, al parecer la profesora no logró realizar una improvisación para reorientar la clase con el propósito de aclarar la falsa idea con la cual finalizaron los estudiantes.

\section{Reflexión-sobre-la acción}

Mary en el proceso de reflexión-sobrela acción, socializa su experiencia en la Comunidad de Práctica, bajo una visión crítica de lo que ha ocurrido en el aula. En primer lugar, comenta lo sucedido en clase:

Cuando yo planeo algo, y miro, y hago y me estrello con que la figura no me queda cerrada en medio de la clase. En el momento pues yo traté de estar lo más tranquila, pero yo por dentro decía: ¿qué fue lo que hice?, cómo fue que me salió esa figura de esa manera que no me di cuenta que no iba a quedar como yo la había planeado. (Episodio 3 - Fragmento de las entrevistas realizadas para este estudio).

Se presenta una situación detonante en la clase que desestabiliza a la profesora, y la obliga a tomar decisiones sobre la marcha de la clase. El hecho es que los estudiantes detectaron detalles que la profesora pasó por alto en la construcción de la figura. Sin lugar a dudas como lo expresa la profesora esta situación la hizo reflexionar sobre la importancia de revisar todas las actividades antes de la clase, más aún cuando se trata de usar tecnologías digitales en clase de matemáticas.

En su reflexión Mary añade en la socialización con sus compañeros que: "Aunque después ya en el resto de la clase al final lo trabajamos y llegamos a la conclusión que sí, el giro tenía que ser $143^{\circ}$ y abajo no podía ser $40^{\circ}$ sino 37" (Episodio 4 - Fragmento de las entrevistas realizadas para este estudio).

Mary ante el hecho no planeado, condujo su clase a partir de la situación surgida y promovió entre sus estudiantes la discusión en búsqueda de la solución. No obstante, la validación de los estudiantes e incluso la de Mary, se basó en el predominio 
de una percepción visual determinada por la imagen del triángulo que se muestra en la pantalla de la computadora (Figura 4) y esta percepción prima sobre el criterio de la suma de los ángulos internos del triángulo antes mencionado. Podemos inferir que nos encontramos ante un triángulo isósceles imposible de construirse bajo los criterios iniciales propuestos en la guía. El proceso de construcción de la figura fue apoyado por una herramienta tecnológica: la misma que permitió a los estudiantes visualizar en la pantalla una figura abierta. Pero las limitaciones del software dificultaron que los estudiantes identificaran una figura imposible de hacer, reafirmando la falsa idea de la construcción de una figura cuya medida de los ángulos son de $70^{\circ}, 37^{\circ}$ y $70^{\circ}$.

Una de las reflexiones finales en Comunidad de Práctica de Mary es:

Las actividades cuando uno las planea, uno quiere que resulten de una manera y en medio de la actividad van saliendo de otra. Me hizo reflexionar sobre mi práctica, sobre todo en la enseñanza de las matemáticas. (Episodio 5 - Fragmento de las entrevistas realizadas para este estudio).

Desde nuestra perspectiva, observamos en el proceso de Reflexión-y-Acción en el interior de la Comunidad de Práctica, transformaciones en las prácticas de los profesores al incorporar las tecnologías digitales. Una evidencia de ellos son las experiencias compartidas por Mary a sus compañeros. Mary enfatiza que, aunque esta actividad fue socializada en comunidad por ella antes de su aplicación, ni ella ni sus compañeros se fijaron en esos detalles que los estudiantes sí identificaron inmediatamente al construir la figura. Esto la motiva a replantear su metodología de revisar y validar el material que introducirá en la clase de matemáticas para disminuir situaciones como la aquí reportada.

\section{Reflexiones finales}

En la marcha de la clase, la profesora Mary trata de dar respuestas a sus estudiantes en búsqueda de la solución al imprevisto gestado. Aunque su pensamiento matemático escolar es claro en el tema en estudio, la misma situación contribuyó para que el propósito de la clase tomara otro cause quedando una sensación de haber superado la dificultad. Se observa que la herramienta hasta cierto punto fue importante en la actividad en su representación gráfica de una figura abierta, esto genera a los estudiantes inquietud por solucionar un problema orientado a cerrar dicha figura. Sin embargo, al final del proceso crea en la pantalla un engaño visual que condujo tanto a los estudiantes como a la profesora a una solución del problema que contradice el teorema de la suma de los ángulos interiores de un triángulo. En este instante consideramos dos aspectos:

- El papel de la herramienta fue insuficiente para verificar la construcción y contribuir con una idea clara al respecto. De aquí la importancia del uso de software como herramienta complementaria, es decir, como se expone en el apartado de reflexión-en-la acción, el uso de diferentes programas dinámicos (Logo y GeoGebra) puede ayudar a la profesora a crear más representaciones alrededor del mismo objeto para validar la solución de un problema. Empero, para ello no solo se requiere cierto dominio de la herramienta sino un dominio desde el pensamiento didáctico y orquestal.

- En el estudio de la geometría se requiere el uso de conceptos figurales, según Fischbein (1993) las figuras geométricas poseen ambos aspectos. Los conceptuales y los figurales.

Los objetos de investigación y manipulación en el razonamiento geométrico son entonces entidades mentales, llamadas por nosotros conceptos figurales, que reflejan propiedades espaciales (forma, posición y tamaño). Al mismo tiempo, poseen cualidades conceptuales, como idealidad, 
abstracción, generalidad y perfección (FISCHBEIN, 1993, p. 143).

Notamos en el caso expuesto una preponderancia de las observaciones de los estudiantes y la profesora basadas en los aspectos figurales, restringiendo así las posibilidades de observar cuáles pueden ser las propiedades necesarias relacionadas con la construcción. Desde nuestra perspectiva, el aspecto conceptual en los estudiantes y la profesora se ve restringido en su aplicación, debido a que no ven la necesidad de apoyarse en las propiedades geométricas al desarrollar sus tareas de construcción y explicación. Se percibe en los estudiantes y en la profesora una separación entre lo conceptual y lo figural.

Aunque dicho material provenga de actividades confiables como en este caso. Mary reafirma la importancia de planear y probar cuidadosamente antes de la clase todos los materiales involucrados en la planeación. Si se tienen dificultades con materiales de buena fuente, qué podría pasar con materiales de dudosa procedencia. También la profesora ve en la planeación un apoyo para conducir la clase, teniendo claro que no siempre lo que se planea resulta. De allí, el pensamiento didáctico de Mary es consecuente con los recursos escogidos para el estudio de los ángulos. Su planeación de clase, la adaptó al contenido matemático escolar teniendo en cuenta las características del grupo y los recursos a su disposición.

Consideramos el detonante que reorientó la clase como una herramienta que permitió promover la reflexión en Comunidad de Práctica sobre el contenido matemático escolar mediado por tecnologías digitales en sus prácticas. Las experiencias de la profesora Mary generó un ambiente de trabajo colaborativo donde se favoreció la comunicación y discusión entre los participantes de la Comunidad de Práctica, las interpretaciones de cada miembro se fundamentaron en prácticas reales en el aula.

\section{Referencias}

BALL, Deborah; HILL, Heather C; BASS, Hyman. Knowing mathematics for teaching: who knows mathematics well enough to teach third grade, and how can we decide? American Federation of Teachers, New Jersey, v. 29, n. 1, p. 14-46, 2005.

CHEVALLARD, Yves; BOSCH, Marianna; GASCÓN, Josep. Estudiar matemáticas: el eslabón perdido entre la enseñanza y el aprendizaje. Barcelona: ICE/Horsori, 1997.

FISCHBEIN, Efraim. The theory of figural concepts. Educational Studies in Mathematics, Netherlands, v. 24, n. 2, p. 139-162, 1993.

GEDDIS, Arthur; WOOD, Eric. Transforming subject matter and managing dilemmas: a case study in teacher education. Teaching and Teacher Education, London, v. 13, n. 6, p. 611-626, 1997.

IBÁÑEZ, Carlos. Un análisis crítico del triángulo pedagógico: una propuesta alternativa. Revista Mexicana de Investigación Educativa. México D.F., v. 12, n. 32, p. 435-456, 2007.

JUNGK, Werner. Conferencias sobre Metodología de la Enseñanza de la Matemática. La Habana: Pueblo y Educación, 1982.

LLINARES, Salvador; KRAINER, Konrad. Mathematics (student) teachers and teacher educators as learners. In: GUTIERREZ, Angel; BOERO, Paolo (Ed.). Handbook of research on the psychology of mathematics education: past, present and future. Netherlands: Sense, 2006. p. 429-459.

PARADA, Sandra. Reflexión sobre la práctica profesional: actividad matemática promovida por el profesor en su salón de clases. 2011. 309 p. Tesis (Doctorado) - Centro de Investigación y Estudios Avanzados del Instituto Politécnico Nacional, México D.F., 2011. 
RIZO, Celia. Investigación sobre la estructuración del curso de geometría de $4 .^{\circ}$ a $6 .^{\circ}$ grados, basada en las transformaciones y la congruencia. Tesis (Doctorado) - Instituto Central de Ciencias Pedagógicas de la República de Cuba. Comisión Nacional de Grados Científicos, La Habana, 1987.

SHULMAN, Lee. Knowledge and teaching: Foundations of the new reform. Harvard educational review, Massachusetts, v. 57 , n. 1, p. 1-23, abr. 1987.

TREFFERS, Adrian; VONK, H. Three dimensions: a model of goal and theory description in mathematics instruction - the Wiskobas Project. Dordrecht: Reidel, 1987.

TROUCHE, Luc. Managing the complexity of human/machine interactions in computerized learning environments: Guiding students' command process through instrumental orchestrations. International Journal of Computers for mathematical learning, Netherlands, v. 9, n. 3, p. 281-307, sept. 2004.

VEGA, Manuel. Introducción a la psicología cognitiva. Madrid: Alianza, 1990.

WENGER, Etienne. Communities of practice: learning, meaning, and identity. Cambridge: Cambridge University Press, 1998.

WOOD, Terry. The international handbook of mathematics teacher education. Rotterdam: Sense, 2008.

Recibido en: 04.06.2015

Aprobado en: 16.02 .2016

Luis Alexander Conde Solano es doctor y magíster en Matemática Educativa del Centro de Investigación y Estudios Avanzados-IPN, México. Es especialista en el desarrollo intelectual y educación de la Universidad Autónoma de Bucaramanga - Fundación Internacional Alberto Merani, Colombia. Es licenciado en Matemáticas y Computación de la Universidad de Pamplona, Colombia.

Sandra Evely Parada Rico es doctora y magister en Matemáticas Educativa del Centro de Investigación y Estudios Avanzados-IPN, México. Es especialista en educación matemática de la Universidad Industrial de Santander, Colombia. Es licenciada en Matemáticas y Computación de la Universidad de Pamplona, Colombia.

Jorge Enrique Fiallo Leal es doctor en Didáctica de las Matemáticas de la Universidad de Valencia, España. Es magister en enseñanza de la Matemática de la Universidad Industrial de Santander, Colombia. Es licenciado en matemáticas de la Universidad Industrial de Santander, Colombia. 